https://doi.org/10.21670/ref.2002044

Artículos

\title{
Cuidado a través de la frontera: trayectorias transfronterizas de cuidados en la región Tijuana-San Diego
}

\section{Caring across borders: transborder care trajectories in the Tijuana-San Diego region}

Silvia López Estrada ${ }^{{ }^{*}}$ (I) https://orcid.org/0000-0002-3241-2806
Recibido el 12 de agosto de 2019. Aceptado el 3 de marzo de 2020. Publicado el 17 de marzo de 2020.

${ }^{*}$ Autora para correspondencia: Silvia López Estrada, correo electrónico slopez@colef.mx

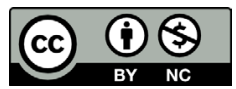

This work is licensed under a Creative Commons Attribution-NonCommercial 4.0 International License. nia, México, correo electrónico: slopez@colef.mx

\section{Resumen}

Este artículo analiza el trabajo transfronterizo de cuidados a través de las trayectorias laborales de mujeres mexicanas residentes de Tijuana que trabajan cuidando niños y adultos mayores y enfermos en San Diego. Debido a que el estudio de las cadenas globales de cuidado ha dado mayor atención a lo transnacional, se enfatiza la dimensión transfronteriza como un elemento novedoso de análisis. Con base en las entrevistas realizadas a un grupo de trabajadoras de cuidados se argumenta que sus trayectorias son transfronterizas debido a que sus arreglos laborales están asociados al cruce de la frontera, en tanto que la rotación laboral está relacionada al curso de vida de las personas a las que se cuida. Asimismo, se encuentra que los elementos que organizan las trayectorias laborales son las motivaciones para el trabajo, el cruce fronterizo, las responsabilidades familiares, el curso de vida y las características individuales de las trabajadoras de cuidados.

Palabras clave: trabajo transfronterizo de cuidados, trabajo remunerado de cuidados, trayectorias laborales transfronterizas, arreglos laborales de cuidado, región Tijuana-San Diego.

\section{Abstract}

This article analyzes the transborder care work trajectories of Mexican women living in the area of Tijuana while working in San Diego, taking care of children and the elderly. Because the study of global care chains focused on the transnational dimension of care activities, the transborder dimension of care work constitutes a novel element for its analysis. Based on the interviews with a group of women, the main argument proposes that their transborder trajectories rely on crossbording as the central aspect that defines care labor arrangements, while turnover results from the life course of the persons who receive care. Besides,

CÓMO CITAR: López, S. (2020). Cuidado a través de la frontera: trayectorias transfronterizas de cuidados en la región Tijuana-San Diego. [Caring across borders: transborder care trajectories in the Tijuana-San Diego region]. Estudios Fronterizos, 21, e044. https:// doi.org/10.21670/ref.2002044 
the elements that organize the trajectories are motivations for care work, family responsibilities, life course, and mobility across the border, as well as women's profile.

Keywords: transborder care work, remunerated care work, transborder labor trajectories, care labor arrangements, Tijuana-San Diego region.

\section{Introducción}

En el contexto de la crisis de los cuidados en el sur y norte globales, y de los factores institucionales que definen las dinámicas de atracción del trabajo transmigrante en Estados Unidos (EUA), en este artículo se analizan las trayectorias laborales transfronterizas de mujeres mexicanas residentes de la ciudad mexicana fronteriza de Tijuana, Baja California, que trabajan en el cuidado de niños y adultos mayores y/o enfermos en la ciudad de San Diego, California, Estados Unidos. Este tipo de actividades remuneradas se refieren al cuidado de niños y adultos mayores, enfermos o dependientes, y forman parte del sector informal de trabajo doméstico en Estados Unidos.

Debido a que la mayoría de los estudios sobre el trabajo remunerado de cuidados en dicho país se refiere a las migrantes desde una perspectiva transnacional, en este artículo se enfatiza el carácter transfronterizo de la actividad, ya que el área fronteriza México-Estados Unidos, por su proximidad geográfica, permite tomar ventaja de obtener un mejor salario en el país vecino mientras se reside en México donde el costo de vida es menor, además de que se tiene la posibilidad de estudiar y/o trabajar, y de participar en el cuidado de la familia. Así lo muestran las narrativas de un grupo de trabajadoras que fueron entrevistadas, y que constituyen la base principal del análisis, el cual se complementa con alguna información de los censos mexicanos.

Las trayectorias laborales y de movilidad transfronteriza de las trabajadoras de cuidados nos permiten analizar sus inicios en la actividad, los cambios en sus arreglos laborales y en sus trayectorias como resultado de las transformaciones en las dinámicas de cruce, las responsabilidades familiares, y factores individuales como la edad, la escolaridad y la experiencia en la actividad.

A la luz de la literatura sobre el tema, en la primera parte de este artículo se discuten los conceptos de cuidados y de trabajo de cuidados. En el contexto más amplio de los cuidados a nivel global, el segundo apartado revisa los estudios sobre el trabajo de cuidados en áreas transfronterizas, y a partir del concepto de transmigración se propone el concepto de trabajo transfronterizo de cuidados, así como el de trayectorias transfronterizas de cuidado. El tercer apartado presenta los aspectos metodológicos, en donde se destaca el uso de las trayectorias como herramienta para el análisis de los casos de estudio. El cuarto apartado, con base en información censal sobre la población transmigrante, analiza algunas características sociodemográficas de las trabajadoras de cuidado, así como el perfil de las que fueron entrevistadas para este estudio. El quinto apartado se centra en el análisis de las trayectorias transfronterizas de cuidados, y las conclusiones resumen los principales hallazgos de este trabajo. 


\section{Los cuidados como trabajo}

El concepto de cuidado ha sido objeto de discusión en las ciencias sociales en las dos últimas décadas. En particular, la crisis de los cuidados ha incentivado la discusión acerca de su definición (Carrasquer, 2013). Desde una perspectiva sociológica destaca la definición de Daly y Lewis (2000), quienes propusieron el concepto de cuidado social como una actividad y un conjunto de relaciones entre diferentes actores sociales, ya que articula el análisis de la vida de las mujeres, los estados de bienestar y sus transformaciones. Para Carrasquer (2013, p. 99), la propuesta de Daly y Lewis intenta sacar el análisis de los cuidados de lo "femenino", trasladando la discusión hacia las necesidades y la provisión de bienestar. Desde esta perspectiva, argumenta Carrasquer, el cuidado es visto como trabajo socialmente organizado ya que participan grupos e instituciones en distintos espacios sociales, y los cuidados se articulan y distribuyen en concordancia con patrones socioculturales y económicos específicos. De acuerdo con la autora, el trabajo femenino es el elemento articulador de la organización social del cuidado que se integra por la familia, el mercado y el Estado, y que conforma regímenes de cuidado. Por último, Carrasquer (2013, p. 100) destaca la importancia de la vida cotidiana en la cual transcurre la vida de las mujeres, y por tanto organiza el trabajo de cuidados entre los distintos actores e instituciones en que se lleva a cabo.

Así, desde la perspectiva de la organización social del cuidado, aquí centramos la atención en el trabajo remunerado de cuidados, cuya contribución es central para la reproducción de la fuerza de trabajo y el desarrollo social (Colen, 1995; Kofman \& Raghuram, 2012; Razavi \& Staab, 2010). Este trabajo involucra a un rango amplio de actividades que son llevadas a cabo en hospitales, casas de retiro, así como en instituciones educativas y profesiones de servicios; además de las actividades reproductivas como el trabajo doméstico y el cuidado informal de los adultos mayores y los niños (Alber \& Drotbohm, 2015). En particular, aquí nos concentramos en el concepto de cuidados como trabajo remunerado que mujeres transmigrantes mexicanas realizan para familias en Estados Unidos, y que se llevan a cabo en el ámbito de los hogares.

En este estudio, el trabajo de cuidados se define como la provisión remunerada de servicios personales para la atención de niños y adultos mayores y/o enfermos en la casa del empleador. Estas actividades son mayoritariamente llevadas a cabo por mujeres e incluyen el cuidado físico, social y afectivo que la trabajadora de cuidados proporciona a las personas que requieren sus servicios en diferentes etapas del curso de vida. Además, el cuidado demanda de tiempo y tiene un carácter relacional entre la persona que cuida y la que recibe sus cuidados (Batthyány, 2010).

\section{La crisis global de los cuidados: de la migración transnacional a la transmigración}

La crisis sistémica que privilegia el capital sobre el bienestar de las personas con impactos en diferentes partes del mundo ha derivado en una crisis de reproducción social tanto en los países del norte global como en los del sur global (Benería, 2008; Pérez-Orozco, 2014). Mientras que en los países del norte hay una demanda de cuidados no calificados y de servicio doméstico debido a la mayor participación económica de las mujeres profesionistas quienes tienen que equilibrar las demandas 
del trabajo y la familia, así como el crecimiento de la población de edad avanzada, y la falta de servicios estatales, en los países del sur la crisis de reproducción social tiene su origen en la pobreza y el desempleo, así como en dinámicas demográficas que movilizan a las mujeres a migrar y trabajar como empleadas domésticas y cuidadoras en los países del norte (Benería, 2008; Hochschild, 2000; Sassen, 2003).

La crisis de cuidados y sus dinámicas de atracción han creado una división internacional del trabajo reproductivo que se transforma a lo largo del tiempo produciendo desigualdades en la intersección de la migración, el género y la etnicidad, en diferentes niveles y dimensiones (Parella, Petroff \& Solé, 2013; Pérez-Orozco, 2014). Al nivel estructural las leyes migratorias y los sistemas de bienestar producen desigualdades afectando el estatus migratorio y la calidad de vida de los individuos; mientras que a nivel microsocial el análisis de las experiencias, subjetividades y agencias de las mujeres ayuda a la comprensión de las formas en que el trabajo de cuidados de las mujeres migrantes está articulado a los procesos económicos globales (Cienfuegos, 2016).

La literatura sobre migración y cuidados destaca el concepto de cadenas globales de cuidados para describir la mercantilización transnacional del trabajo de cuidados que resulta de la migración de trabajadoras domésticas y de cuidados de los países del sur a los países del norte (Hochschild, 2000; Sassen, 2003; Yeates, 2005), donde las migrantes transfieren su trabajo en los países de destino mientras que delegan a otras las actividades de cuidado en sus países de origen (Hochschild, 2000).

Yeates (2009) y Kofman y Raghuram (2012) argumentan que las cadenas globales, como perspectiva transnacional, limitaron el análisis a ciertas regiones geográficas (sur a norte), instituciones y regímenes de cuidado, al destacar la importancia de poner atención a las desigualdades de género y etnicidad en regiones específicas. Investigaciones recientes en Europa y América Latina ya han tomado en cuenta otras geografías del trabajo de cuidados a nivel global, como es el caso de las migraciones sur-sur (Maher, 2003; Durin, De la O \& Bastos, 2014), destacando la participación de las migrantes como "trabajadoras reproductivas" (Truong, 1996) dedicadas al cuidado de las familias en los países de destino (Carcedo, Chávez \& Lexartza, 2011; Mattingly, 2006; Pérez-Orozco, 2014; Soto, González \& Dobrée, 2012).

A pesar de lo anterior, menor atención se ha dado a la dimensión transfronteriza del trabajo de cuidados, de ahí el interés de este artículo por analizar este tipo de trabajo en una de las regiones más transitadas del mundo, como es el área fronteriza de Tijuana con San Diego, que se caracteriza por su población transmigrante.

\section{La transmigración y el trabajo de cuidados}

Estudios recientes, como el de Leiva y Orellana (2016), sobre el trabajo de cuidados en algunas regiones fronterizas analizan esta actividad a partir del concepto de migración circular. Estos autores destacan investigaciones de cuidadoras circulares de Polonia y Ucrania a Italia (Marchetti, 2013), de mujeres polacas hacia Alemania (PalengaMöllenbeck, 2013), migración de rumanas a España (Marcu, 2009). En América Latina, Leiva y Orellana (2016) y Leiva, Mansilla y Comelin (2017) estudiaron la migración circular de bolivianas en Chile. Los autores parten del concepto de migración circular enfatizando la condición fronteriza de migrantes que se desplazan a otro país de 
manera temporal, ya que no tienen la intención de residencia a largo plazo (Leiva y Orellana, 2016).

A diferencia del estudio anterior, aquí recuperamos el concepto de transmigración que da cuenta del ir y venir de las personas a través de la frontera México-Estados Unidos. Los transmigrantes son personas que viven en las ciudades fronterizas del norte de México como Tijuana, y cruzan la frontera en forma periódica para trabajar en las ciudades vecinas del sur de Estados Unidos (Alegría, 2002). Mientras que los migrantes internacionales cambian de residencia, la población transmigrante va a Estados Unidos por periodos cortos de tiempo, de un día a una semana o un mes, para después regresar a México (Orraca, 2015, p. 1, citado en López, 2019).

En la región fronteriza de México con Estados Unidos, las mexicanas migrantes en EUA. han participado en actividades de cuidado desde hace tiempo como apoyo a las mujeres nativas profesionistas (Ehrenreich \& Hochschild, 2003). Este fenómeno se ha modificado como producto de transformaciones económicas, la migración y las políticas de bienestar en Estados Unidos. Dicho patrón de empleo inició a principios del siglo xx, y se volvió más común en los años de 1940 cuando las mujeres mexicanas empezaron a sustituir a las afroamericanas en este tipo de trabajos (Ehrenreich \& Hochschild, 2003; Maher, 2003). El fenómeno se incrementó en las clases medias a partir de la década de 1980 con un número creciente de hogares en California que empleaban a las mujeres migrantes mexicanas como nanas y trabajadoras domésticas (Maher, 2003, p. 6; Mattingly, 2006, p. 111).

Los estudios de Hondagneu-Sotelo (2007, p. 28), Pisani y Yoskowitz (2002), y Mattingly (2006) asociaron el trabajo transfronterizo de cuidados al estado civil y el estatus legal de las mujeres en Estados Unidos. Así, mientras que las trabajadoras en arreglos live-in eran solteras, residían con la familia y estaban disponibles todo el tiempo, pero carecían de documentos legales y tenían menos experiencia, razón por la cual ganaban menos. En cambio, las trabajadoras con arreglo live-out, eran casadas, educadas y con mayor experiencia, además de que tenían documentos legales y mejores salarios, y podían trabajar con distintas familias. Esta modalidad de trabajo les permitía cruzar la frontera de regreso a casa para ver a sus propias familias y atender sus responsabilidades domésticas. Como se muestra más adelante con los casos de estudio, además del estatus legal, al parecer la participación de las mujeres transfronterizas en estas modalidades de trabajo se ha modificado a partir de la menor intensidad en el cruce de la frontera. De acuerdo con Coubès (2008), las crecientes dificultades para cruzar la frontera tienen implicaciones para la menor o mayor participación de las mujeres en el trabajo de cuidados.

Por último, la literatura también destaca el uso de las redes sociales informales para conseguir trabajo de cuidados de tiempo completo y de largo alcance (HondagneuSotelo, 2007; Maher, 2003; Mattingly, 2006), así como el trato que se les da a las empleadas domésticas, el cual al parecer depende de su estatus legal de trabajo (Mattingly, 2006).

\section{Las trayectorias transfronterizas de cuidados}

A través del estudio sistemático de los cambios en la transmigración y el empleo se pueden conocer los cambios en la movilidad laboral y espacial (Rivera, 2012, p. $460)$, así como los factores que intervienen y la relación entre los mismos. De manera 
particular, se revelan las experiencias de las personas, sus interpretaciones sobre los cambios y los significados que estos tienen para ellas, a partir del itinerario subjetivo como herramienta conceptual que combina elementos temporales con los cambios en la movilidad geográfica, y las emociones y sentido de pertenencia desarrollados a través de la experiencia migratoria (Hirai \& Sandoval, 2016, p. 285).

Aquí usamos las trayectorias como una herramienta analítica para la comprensión de las historias de trabajo y trayectos profesionales. En este caso el análisis tiene el objetivo de observar el ir y venir de las trabajadoras de cuidados a través de la frontera, en dimensiones de movilidad laboral y socioespacial.

Aunque en los estudios sobre migración y cuidados aparecen pocas referencias a las trayectorias laborales, Leiva y Orellana (2016) caracterizan las trayectorias de mujeres bolivianas que realizan trabajo de cuidados en Chile por su hiperfragmentación que consiste en trabajos de corta duración, largas jornadas, a veces sin pago y alta vulneración de derechos. Para los autores son las condiciones de trabajo precario lo que ocasiona la alta fragmentación de las trayectorias de las mujeres que entrevistaron. En general, la precariedad es característica del trabajo de cuidados (en un texto anterior analizamos los rasgos de precariedad del grupo de trabajadoras de cuidado entrevistadas). Sus contratos eran de palabra, las condiciones laborales eran variables y a discreción de las patronas, los salarios por hora eran menores al salario legal, y no tenían acceso a la seguridad social (López, 2019).

Por su parte, Cretu (2017) analiza las trayectorias transfronterizas de mujeres profesionistas de países postsoviéticos en Londres. El estudio define las trayectorias transfronterizas como movilidad entre ciudades (in-between-cities mobility), y la autora propone usar las trayectorias para comprender por qué las mujeres construyen trayectorias transfronterizas, y delinear las similitudes que existen en sus carreras en el contexto de procesos sociales que les dan forma (Cretu, 2017, p. 62).

En este estudio, las trayectorias transfronterizas de cuidados se definen como los trayectos laborales de las trabajadoras que implican movimientos de periodos cortos de tiempo a través de la frontera Tijuana-San Diego para trabajar en esa ciudad mientras que residen, trabajan y/o estudian en el área metropolitana de Tijuana y en Ensenada. Las trayectorias de cuidados de las entrevistadas observan diferentes arreglos laborales que están asociados a las dinámicas de cruce, y que refieren a la dimensión socioespacial a la escala del hogar: live-in, live-out. Además, del estudio de Cretu recuperamos las responsabilidades familiares y el curso de vida como elementos de análisis de las trayectorias.

\section{Metodología}

La mayoría de los estudios llevados a cabo en algunas ciudades fronterizas de Estados Unidos y México centran la atención al trabajo de las migrantes residentes en EuA. Por esta razón aquí privilegiamos la transmigración de mujeres mexicanas que viven en Tijuana y cruzan la frontera para trabajar en San Diego como cuidadoras de niños, adultos mayores y enfermos.

De carácter cualitativo, este estudio se basa en las experiencias de trabajo transfronterizo de cuidados de un grupo de mujeres entrevistadas para analizar las dinámicas e interacciones sociales que tienen como eje esta actividad, a partir de sus trayectorias laborales. 
Para ello, en 2016 se llevaron a cabo entrevistas con seis trabajadoras de cuidados que fueron contactadas a través de la técnica de bola de nieve, y una más que fue entrevistada en 2018. Los contactos se llevaron a cabo a través de colegas y amistades que conocían a estas trabajadoras. Cinco entrevistas fueron llevadas a cabo en Ensenada, Playas de Rosarito y Tijuana, y dos en San Diego. En ningún caso las mujeres accedieron a ser entrevistadas en sus casas, por lo cual las entrevistas se llevaron a cabo en lugares públicos como cafés y restaurantes, así como en un centro comercial.

La mayoría de las mujeres accedieron a que la entrevista fuera grabada, y cuando no fue así se tomaron notas. A todas se les entregó una carta de consentimiento informado, pero no aceptaron firmarla porque se sienten en riesgo o suponen algún tipo de compromiso. Se les explicó que podían solicitar información sobre el proyecto, que podían suspender la entrevista si en algún momento les incomodaba, y que sus nombres no serían mencionados para preservar su privacidad. Una segunda etapa de campo se inició en 2018, para lo cual se contactó a varias trabajadoras que resultaron ser mayores de 40 años, unas se dedicaban a cuidar niños y otras a adultos mayores. Sin embargo, por este tiempo llegó a Tijuana La Caravana Migrante, lo que creó un contexto de riesgo para los transmigrantes, ya que muchos de ellos cruzan a trabajar en Estados Unidos con su visa de turista. Debido a ello, las trabajadoras de cuidados que habían sido contactadas se negaron a ser entrevistadas pues temían ser deportadas. Su preocupación era totalmente justificada, ya que la amenaza del gobierno de Estados Unidos de cerrar la frontera creó incertidumbre no solo para el comercio y el turismo, sino para la gente que vive en Tijuana y depende del cruce cotidiano de la frontera para ir a trabajar a San Diego. En el verano de 2019 se llevó a cabo otro bloque de entrevistas, sin embargo, debido a cuestiones de tiempo ya no fue posible integrarlas a este texto.

La información de las entrevistas fue sistematizada a partir de algunas categorías que emergieron de los relatos, según el método de la teoría fundamentada (Glaser \& Strauss, 2012), y se realizó un análisis interpretativo. En forma complementaria a las narrativas de las trabajadoras entrevistadas, también usamos información censal e intercensal de México, obtenida de la base de datos Integrated Public Use Microdata Series del Minnesota Population Center (2017), para describir algunos rasgos del perfil de las trabajadoras de cuidados como parte de la población transmigrante.

\section{Transmigración y trabajo de cuidados en la frontera Tijuana-San Diego}

Para algunos autores, la región Tijuana-San Diego funciona como una metrópoli transfronteriza que cumple funciones complementarias. Así, mientras San Diego se especializa en actividades de alta tecnología, Tijuana está orientada hacia servicios y manufactura intensiva con mano de obra poco calificada. Estas ciudades forman parte de dos distintos países que se caracterizan por significativas diferencias y asimetrías económicas políticas y culturales (Alegría, 2002).

A pesar de las diferencias en ingresos, infraestructura, lenguaje y cultura (Mendoza, 2017), miles de personas cruzan estas fronteras en forma cotidiana, siendo Tijuana-San Ysidro una de las fronteras internacionales más cruzadas del mundo. Entre los cruces cotidianos destacan los de las personas que viven en Tijuana y trabajan en San Diego. Alegría (2002) llamo a este proceso transmigración y está basado en la proximidad 
geográfica y las actividades económicas relacionadas de las ciudades en ambos lados de la frontera.

La transmigración de México a Estados Unidos es principalmente de tipo económica. Las trabajadoras transfronterizas de cuidados forman parte de la población transmigrante de las ciudades fronterizas, su trabajo es parte del sector informal del servicio doméstico en Estados Unidos. A pesar de la existencia de leyes laborales e impositivas (Mattingly, 2006), se trata de un mercado de trabajo flexible y desregulado, teniendo como resultado una ocupación precaria. Además, el estatus migratorio, en combinación con el origen étnico y el género tiene efectos en los bajos salarios, la inestabilidad y falta de protección social de estas trabajadoras (Maher, 2003; Hondagneu-Sotelo, 2007; Williams, 2011; Ariza, 2011).

Es difícil obtener estadísticas acerca de los trabajadores transmigrantes, pero el censo mexicano incluye alguna información al respecto. Así, con base en datos de los censos en México, estudios recientes muestran que la población transmigrante en las ciudades fronterizas del norte de México está descendiendo, por ejemplo, en Tijuana, este grupo bajó su porcentaje de 5.6\% a 4.5\% entre 2000 y 2010 (Orraca, 2015; Vargas \& Coubès, 2017, citados en López, 2019).

A pesar de lo anterior, las mujeres transmigrantes se incrementaron de $23 \%$ a $30 \%$ en ese mismo periodo (Orraca, 2015). Aunque en el censo no existe el concepto de trabajo transfronterizo de cuidados, es posible acercarse a estas actividades a través de la categoría de "ocupaciones elementales", y $31.7 \%$ de las mujeres transmigrantes se concentran en esa categoría (Orraca, 2015, citado en López, 2019, p. 125). Un análisis más detallado de la información censal reveló que se trata de mujeres que se dedican al trabajo doméstico y de cuidado, y que su número se incrementó más de $100 \%$ entre 2000 y 2010. Es posible que lo anterior se deba a que, durante la crisis económica mundial de 2008, la tasa de desempleo se incrementó de 0 a $8 \%$ por primera vez en 20 años (Coubès \& Silva, 2013), y debido al desempleo de sus parejas, muchas amas de casa tuvieron que buscar empleo, por lo que el trabajo de cuidados en San Diego resultó una opción para ellas.

Los datos del censo sugieren que más mujeres jóvenes y educadas están participando en esta actividad, mientras que en 2000 la edad promedio de las trabajadoras transfronterizas de cuidados era de 40.2 años, en 2015 el número de mujeres jóvenes tuvo un notable aumento, de $16.7 \%$ a $47.6 \%$, y por tanto hubo un descenso de la edad promedio, de 52.5 en 2010 a 40.6 en 2015 (véase Tabla 1).

Tabla 1: Trabajadoras mexicanas transfronterizas de cuidados en San Diego por grupo de edad, 2000, 2010, 2015

\begin{tabular}{|c|c|c|c|c|c|c|}
\hline Grupo de edad & 2000 & $\%$ & 2010 & $\%$ & 2015 & $\%$ \\
\hline Menos de 30 & 77 & 16.7 & 59 & 7.5 & 504 & 47.2 \\
\hline Más de 30 & 382 & 83.2 & 724 & 92.5 & 554 & 53.3 \\
\hline Total & 459 & 100.0 & 783 & 100.0 & 1058 & 100.0 \\
\hline
\end{tabular}

Fuente: elaboración propia con base en el Censo General de Población y Vivienda 2000, Censo de Población y Vivienda 2010 y Encuesta Intercensal 2015, de México, obtenidos a través de Minnesota Population Center (2017). 
Según la Tabla 2 el perfil educativo de este grupo de trabajadoras de cuidados ha ido cambiando a través del tiempo, mientras que en 2000 pocas de ellas iban a la escuela preparatoria, $15 \%$ sí asistía en 2015 . Este incremento en la escolaridad parece estar asociado a la edad, ya que en la medida en que se incorporan más mujeres jóvenes se incrementa la posibilidad de que se encuentren estudiando.

\begin{tabular}{|c|c|c|c|}
\hline & 2000 & 2010 & 2015 \\
\hline Edad promedio & 40.2 & 52.5 & 40.6 \\
\hline $\begin{array}{l}\text { Porcentaje de mujeres con } \\
\text { preparatoria }\end{array}$ & 0.0 & 0.0 & $13.8 \%$ \\
\hline Promedio de salarios & 6746 & 6402 & 11060 \\
\hline
\end{tabular}

Fuente: elaboración propia con base en el Censo General de Población y Vivienda 2000, Censo de Población y Vivienda 2010 y Encuesta Intercensal 2015, de México, obtenidos a través de Minnesota Population Center (2017).

Entre 2000 y 2010 el ingreso promedio de las trabajadoras disminuyó de 6746 a 6402 dólares, mientras que aumentó a 11060 dólares en 2015. En ese año, el ingreso máximo registrado fue de 24000 dólares, menor que el costo promedio nacional (28 964) de contratar a una nana en Estados Unidos en 2015 (Schulman \& Blank, 2017). De acuerdo con la misma fuente, California se distingue como el estado con el costo más alto por este servicio de cuidados.

\section{El perfil de las trabajadoras transfronterizas de cuidados entrevistadas}

La Tabla 3 muestra el perfil sociodemográfico de las entrevistadas. Tres de las mujeres tienen menos de 40 años (jóvenes), y cuatro son mayores de 60 (adultas). Las mujeres de más edad provienen de otros estados como Jalisco, Oaxaca y Michoacán; en tanto que dos de las jóvenes nacieron en Tijuana y una en San Diego. En relación con el estado civil, cinco eran casadas, una estaba separada de su pareja y la otra era viuda. Susy, Andrea y Martha son las más jóvenes del grupo; cuando se iniciaron en la actividad eran solteras, al momento de la entrevista Susy y Andrea estaban casadas y Martha se había comprometido. En cuanto a su escolaridad, mientras que dos de las jóvenes tenían educación profesional, una de ellas dejó la universidad cuando cursaba el segundo año; por su parte, las mujeres mayores tenían educación primaria y media. 
Tabla 3: Características sociodemográficas de las entrevistadas

\begin{tabular}{|c|c|c|c|c|c|c|c|c|c|}
\hline & Edad & $\begin{array}{c}\text { Estado } \\
\text { civil }\end{array}$ & $\begin{array}{c}\text { Hijos } \\
1-6 \\
\text { años }\end{array}$ & $\begin{array}{l}\text { Nivel de } \\
\text { educación }\end{array}$ & $\begin{array}{c}\text { Estatus } \\
\text { migratorio }\end{array}$ & $\begin{array}{c}\text { Años } \\
\text { en } \\
\text { trabajo } \\
\text { de } \\
\text { cuidados }\end{array}$ & $\begin{array}{l}\text { Arreglo } \\
\text { laboral }\end{array}$ & $\begin{array}{l}\text { Salario } \\
\text { semanal }\end{array}$ & $\begin{array}{c}\text { Protección } \\
\text { Social en } \\
\text { Tijuana }\end{array}$ \\
\hline Susy & 28 & Casada & & $\begin{array}{l}\text { Universitaria } \\
\text { inconclusa }\end{array}$ & Visa turista & 5 & Live-in & 400 & Sí \\
\hline Andrea & 30 & Casada & & Universitaria & Ciudadana & 15 & Live-in & $20 \mathrm{x}$ hora & Sí \\
\hline Martha & 28 & Casada & & Universitaria & Visa residente & 8 & Live-out & 450 & No \\
\hline Matty & 63 & Casada & & Secundaria & Indocumentada & 8 & Live-in & 300 & No \\
\hline Teresa & 40 & Casada & 1 & Preparatoria & Visa turista & 4 & Live-in & 350 & Sí \\
\hline Dolores & 55 & Separada & & Preparatoria & Visa turista & 2 & Live-in & 350 & Sí \\
\hline Rita & 61 & Viuda & & Primaria & Visa turista & 4.5 & Live-in & 425 & No \\
\hline
\end{tabular}

Fuente: elaboración propia a partir de entrevistas realizadas en 2016. Todos los nombres de las entrevistadas han sido modificados para preservar su identidad.

Con la excepción de una trabajadora, los salarios de las entrevistadas eran variables y menores al mínimo legal en California en 2016 (10.50 dólares por hora), y al parecer estaban relacionados con su edad, educación y experiencia, tal como lo muestra la Tabla 3. Ninguna de ellas tenía protección social, aunque algunas tenían beneficios sociales en México porque tenían otro empleo ahí o debido al empleo del esposo.

\section{Dinámicas de las trayectorias laborales transfronterizas: arreglos laborales, responsabilidades familiares y patrones de movilidad}

Las trayectorias laborales transfronterizas se refieren a las modalidades de trabajo de cuidados, así como a la organización de la misma a lo largo del tiempo en relación con las responsabilidades familiares, la movilidad a través de la frontera y factores de tipo individual. En un trabajo anterior advertimos que este grupo de entrevistadas se diferencia por la edad y la escolaridad (López, 2019), de esta forma las experiencias de las mujeres reflejan configuraciones en donde estos elementos, además del estatus migratorio dan ventaja a unas sobre otras.

Las trabajadoras de cuidados entrevistadas eran residentes de Tijuana y trabajaban en San Diego cuidando a niños o personas dependientes. De ellas, tres iniciaron como niñeras en San Diego cuando eran estudiantes, así que por años combinaron el trabajo y el estudio, aunque esta situación se modificó con el tiempo. Para ellas participar en esta actividad fue un medio para continuar sus estudios en Tijuana pero, como se muestra más adelante, sus experiencias siguieron diferentes trayectos.

Las mujeres mayores de 40 años se iniciaron como cuidadoras transfronterizas después del matrimonio y la crianza de los hijos debido a que deseaban mejorar sus ingresos como Matty, Rita y Teresa. Dolores, principal sostén de su familia, trabajó por 20 años en una tienda de ropa en San Diego, pero debido al creciente cierre de la frontera en 2016 decidió dejar ese empleo y buscó trabajo como niñera porque pensó que se sentiría más segura trabajando dentro de una casa. 


\section{Responsabilidades familiares}

Las responsabilidades familiares de las entrevistadas se modifican a través de su curso de vida, generalmente estas se incrementan con el matrimonio y la crianza de los hijos, por lo que las trabajadoras llevaban a cabo diferentes arreglos en sus familias a fin de poder trabajar cuidando niños y adultos mayores en San Diego.

Matty, Teresa, Dolores y Rita componen el grupo de mujeres de mayor edad, y con excepción de Teresa, quién tenía un hijo de 8 años a quien dejaba a cargo de su esposo, el resto de las mujeres empezaron a trabajar cuidando niños y adultos mayores cuando sus hijos ya se habían independizado o eran estudiantes, y no requerían el mismo cuidado que cuando eran pequeños. Por esta razón sus responsabilidades familiares eran menores y tenían menos restricciones de tiempo para trabajar del otro lado de la frontera.

De las trabajadoras jóvenes, Andrea vivía con su familia, Susy estaba casada pero no tenía hijos y su esposo se hacía cargo de la limpieza de su departamento, en tanto que Martha vivía con su familia cuando era joven, y al igual que Andrea contribuía a las tareas del hogar, y cuando se fue a vivir a San Diego dividía el trabajo doméstico con su pareja.

\section{Arreglos laborales y (dis)continuidad en la trayectoria}

Como ya mencionamos, Pisani y Yoskowitz (2002) y Mattingly (2006) encontraron que las trabajadoras que se quedaban en casa (live-in) lo hacían porque no tenían documentos, y que las que sí los tenían trabajaban de entrada y salida (live-out), por lo general estaban casadas, por lo cual preferían regresar con sus familias en México.

Las trayectorias de las entrevistadas para este estudio muestran que los arreglos laborales se han modificado con el tiempo debido a que cada vez es más difícil cruzar la frontera. Por ello, los arreglos live-in implican que las mujeres se quedan con la familia con la que trabajan varios días de la semana y regresan a sus casas en Tijuana durante el fin de semana. Todas recibían hospedaje y comida en casa de sus patronas, en forma adicional a sus salarios. En cambio, solo Martha tenía un arreglo live-out, ella inicialmente regresaba a su casa en Tijuana, pero cuando solicitó la residencia en Estados Unidos tuvo que quedarse a vivir en San Diego con su pareja.

En el contexto de una frontera cada vez más cerrada debido a las políticas migratorias de Estados Unidos, los tiempos de cruce se han alargado, lo cual ha tenido consecuencias para las dinámicas de movilidad y trabajo de las entrevistadas que se han transformado a través del tiempo. Por ejemplo, las entrevistadas más jóvenes se iniciaron en la actividad de cuidar niños o enfermos cuando eran estudiantes, y sus arreglos laborales se modificaron cuando pasaron de trabajar los fines de semana a trabajar de lunes a viernes. Este fue el caso de Susy, quien se inició como trabajadora de cuidados atendiendo a un enfermo durante el verano y los fines de semana, al mismo tiempo que estudiaba una carrera universitaria y también trabajaba largas jornadas en un cinema en Tijuana (y otros trabajos de menor valía). Ella sentía que era explotada en estos empleos y decidió dejarlos, así como también interrumpió sus estudios y se fue a trabajar a San Diego como niñera, aunque seguía viviendo en Ensenada con su esposo a quien veía solo los fines de semana. 
Martha, por su parte, también trabajó como niñera desde muy joven con el objetivo de pagar sus estudios. Sin embargo, al terminar su carrera como dentista solo obtuvo un empleo como asistente dental en un consultorio dental de Tijuana, de ahí que decidió trabajar nuevamente como niñera de tiempo completo en San Diego con la idea de establecer algún día su propio consultorio. Así que ambas cruzaban la frontera a San Diego el domingo para volver a Tijuana los viernes en la tarde. Andrea, por su parte, inició trabajando los fines de semana, y con el tiempo fue modificando sus horarios laborales, ya que alternaba este trabajo con sus estudios y otros empleos en Tijuana.

Algunas entrevistadas hablaron de experiencias gratas de buen trato y mejores condiciones laborales, y un par de ellas comentó que en el inicio de sus trayectorias se cambiaron varias veces de trabajo debido a condiciones de maltrato y explotación; cuando inician suelen conseguir trabajos de entrada por salida (live-out), trabajando con distintas familias, hasta que logran encontrar un trabajo más estable.

Los casos de explotación laboral coinciden con los hallazgos de Leiva y Orellana (2016) que argumentan fragmentación de la trayectoria debido al maltrato de las trabajadoras. Sin embargo, en un análisis previo encontramos que cuando las trabajadoras de cuidado lograron un trabajo más estable, la rotación laboral parecía estar asociada al curso de vida de las personas a las que cuidaban, por ejemplo, cuando los niños crecían y se iban al high school (preparatoria), y en el caso de los enfermos el trabajo terminaba cuando el paciente fallecía (López, 2019, p. 133).

De esta forma, cuando las mujeres logran obtener un trabajo estable con una sola familia cuatro o cinco días a la semana, con la que residen (live-in) y cruzan la frontera los lunes y regresan a Tijuana los viernes, ellas hacen lo posible por conservarlo pues saben que les será difícil conseguir otro en las mismas condiciones.

\section{Patrones de movilidad}

Las trayectorias transfronterizas de las trabajadoras de cuidados reflejan también los patrones de movilidad a través de la frontera, los cuales son definidos por su estatus migratorio y las actividades que llevan a cabo en ambos lados de la frontera. La mayoría de las trabajadoras de cuidados cruzaba con una visa de turista, sin embargo, esto puede cambiar con el tiempo ya que algunas mujeres pueden obtener su residencia (visa de residente o green card) como fue el caso de Martha. De acuerdo con el sitio electrónico del gobierno de los Estados Unidos, la visa de residente o green card concede el estatus de residencia permanente a los inmigrantes para vivir y trabajar permanentemente en Estados Unidos. En la mayoría de los casos la visa se solicita por un empleador o familiar en favor de otra persona. La pareja de Martha solicitó su residencia permanente, por esa razón ella tuvo que ir a residir a San Diego hasta que se resolviera su estatus.

En el caso de la visa de turista, esta tiene que renovarse cada determinado tiempo, pero se corre el riesgo de que al renovarla las autoridades de migración se den cuenta de qué persona está trabajando en San Diego y entonces le retira la visa, como fue el caso de Matty, quien al no renovar su visa se convirtió en indocumentada. 


\section{Las características individuales}

La educación formal y la experiencia en el trabajo de cuidado de niños y adultos mayores y dependientes parecen ser los factores que tienen efectos positivos en las trayectorias de cuidados de las entrevistadas, tanto las jóvenes como las adultas consideran que han tenido un progreso salarial y algunas condiciones de trabajo gracias a su experiencia.

La edad es un importante predictor de movilidad laboral en trabajadoras migrantes latinoamericanas en otros países (Parella, Pettrof \& Solé, 2013), ya que a más edad hay menos posibilidades de encontrar empleo y de movilidad laboral. Aunque los casos de estudio parecen coincidir con este patrón, ya que las mujeres adultas entrevistadas tuvieron más dificultades para conseguir trabajo, así como para negociar sus salarios, también es importante considerar la experiencia, pues en la medida en que las mujeres han tenido varios trabajos se vuelven más experimentadas y confiables para sus patronas, con posibilidades de negociar mejores salarios y condiciones laborales.

En conclusión, las trayectorias transfronterizas de las trabajadoras entrevistadas se definen por el tipo de actividad que desempeñan — cuidado de adultos mayores y dependientes o cuidado infantil-, el tipo de arreglo laboral (live-in, live-out), el perfil sociodemográfico (edad, estado civil y educación), y la movilidad laboral asociada al patrón de movilidad transfronteriza. A continuación, describimos con mayor detalle dos de las trayectorias que muestran en contraste las experiencias de dos mujeres con distintos perfiles sociodemográficos y arreglos laborales, una dedicada al cuidado de enfermos y otra al cuidado de niños.

\section{a) Trabajar y estudiar en Tijuana, trabajar en San Diego}

Este tipo de trayectoria parece ser propia de mujeres jóvenes que trabajaban como niñeras o cuidando adultos mayores en San Diego durante los fines de semana, mientras estudiaban la preparatoria o profesional y/o trabajaban en Tijuana en ocupaciones manuales y profesionales. Un rasgo que también se advierte en estas trayectorias es que el patrón de trabajo y movilidad se modificó, ya que a veces al terminar sus estudios universitarios continuaron en el trabajo de cuidados. Aquí se ilustra el caso de Andrea quien trabajaba en el cuidado de pacientes con enfermedades degenerativas y terminales. Para ella el trabajo de cuidados es su oficio, o lo que ella denomina como "profesión de vida", el cual aprendió a través de su familia, y fue el medio para su seguridad económica y el logro de sus objetivos profesionales.

Andrea residía en Playas de Rosarito $^{1}$ al momento de la entrevista, joven, con educación profesional y amplia experiencia, ya que trabajó cuidando enfermos durante 15 años, permaneció tres años en su último trabajo. Aunque ella es ciudadana estadounidense, siempre vivió en Playas de Rosarito con su papá y sus hermanas, mientras que su mamá vivía en Estados Unidos. Se inició en la actividad de cuidados desde que era estudiante de preparatoria, cuando sus tías que eran enfermeras en San Diego la invitaron a trabajar con ellas cuidando a un enfermo. Andrea se ha dedicado a atender personas que padecen enfermedades degenerativas o terminales que

\footnotetext{
${ }^{1}$ El municipio de Playas de Rosarito forma parte de la zona metropolitana de Tijuana, y se localiza a 20 kilómetros de esta ciudad.
} 
requieren atención 24 horas, para ello, junto con sus tías y primas, formaba equipos de trabajo compuestos de dos o tres personas que tomaban turnos de trabajo de 48 o 72 horas, a veces de una semana por una semana de descanso.

Tabla 4: Trayectoria transfronteriza de trabajo de cuidados. Andrea, 30 años, maestría en comunicación, cuida adultos mayores y enfermos

\begin{tabular}{|c|c|c|c|c|c|}
\hline Año & Ciudad & Ocupación & $\begin{array}{c}\text { Horario de } \\
\text { trabajo }\end{array}$ & Salario & $\begin{array}{c}\text { Estatus } \\
\text { migratorio } \\
\text { y cruce }\end{array}$ \\
\hline 2001-2007 & San Diego & $\begin{array}{c}\text { Paciente con } \\
\text { Alzheimer }\end{array}$ & $\begin{array}{c}\text { Fines de } \\
\text { semana }\end{array}$ & 10 dls. $x$ hora & $\begin{array}{l}\text { Ciudadana. } \\
\text { Va y viene a } \\
\text { través de la } \\
\text { frontera }\end{array}$ \\
\hline $\begin{array}{r}2001-2004 \\
2004-2008 \\
2008-2009\end{array}$ & Tijuana & $\begin{array}{c}\text { Estudia la } \\
\text { preparatoria } \\
\text { Estudia en la } \\
\text { universidad } \\
\text { Becaria de } \\
\text { investigación }\end{array}$ & Lun-vie & & \\
\hline 2007-2009 & San Diego & $\begin{array}{l}\text { Paciente con } \\
\text { trombosis }\end{array}$ & $\begin{array}{c}\text { Fines de } \\
\text { semana }\end{array}$ & 15 dls. $\mathrm{x}$ hora & $\begin{array}{l}\text { Ciudadana. } \\
\text { Va y viene a } \\
\text { través de la } \\
\text { frontera }\end{array}$ \\
\hline 2010 & San Diego & Diabético & Lun-vie & & \\
\hline 2010 & San Diego & Anorexia & $\begin{array}{c}\text { Fines de } \\
\text { semana }\end{array}$ & 15 dls. $\mathrm{x}$ hora & \\
\hline 2010 & Tijuana & $\begin{array}{c}\text { Profesora } \\
\text { universitaria }\end{array}$ & Flexible & Variable & \\
\hline 2012 & San Diego & $\begin{array}{l}\text { Paciente con } \\
\text { cáncer }\end{array}$ & $\begin{array}{c}9-5 \\
\text { Lun-vie }\end{array}$ & 15 dls. $x$ hora & $\begin{array}{l}\text { Ciudadana. } \\
\text { Va y viene a } \\
\text { través de la } \\
\text { frontera }\end{array}$ \\
\hline 2013 & San Diego & $\begin{array}{l}\text { Paciente con } \\
\text { cáncer }\end{array}$ & $\begin{array}{c}\text { Fines de } \\
\text { semana }\end{array}$ & 20 dls. $x$ hora & \\
\hline 2013 & San Diego & $\begin{array}{l}\text { Paciente } \\
\text { diabético }\end{array}$ & $\begin{array}{c}\text { Fines de } \\
\text { semana }\end{array}$ & 20 dls. $x$ hora & \\
\hline 2013 & Tijuana & $\begin{array}{c}\text { Profesora } \\
\text { universitaria }\end{array}$ & Flexible & Variable & \\
\hline $2013-2014$ & España & $\begin{array}{l}\text { Estudiante de } \\
\text { maestría }\end{array}$ & & & \\
\hline $2014-2016$ & San Diego & $\begin{array}{c}\text { Paciente con } \\
\text { Alzheimer }\end{array}$ & Lun-vie & 20 dls. $x$ hora & $\begin{array}{l}\text { Ciudadana. } \\
\text { Va y viene a } \\
\text { través de la } \\
\text { frontera }\end{array}$ \\
\hline
\end{tabular}

Fuente: elaboración propia con base en entrevista realizada en 2016.

Ser ciudadana de Estados Unidos le permitía a Andrea el libre tránsito por la frontera con una estrategia combinada de trabajar en los cuidados de adultos mayores 
durante los fines de semana en San Diego, y trabajar en actividades académicas durante los días de la semana en Tijuana. Mientras era estudiante, Andrea cruzaba la frontera los fines de semana para atender al paciente en turno y regresaba a Tijuana el domingo para continuar sus estudios en la universidad o atender su trabajo como becaria de investigación en un centro público y, después, al graduarse, como maestra universitaria. Incluso, cuando aún era estudiante decidió darse de baja un semestre para dedicarse a cuidar de tiempo completo a uno de sus pacientes. Después ella consiguió una beca para continuar sus estudios de posgrado en España, pero a su regreso a Playas de Rosarito continuó trabajando en el cuidado de enfermos en San Diego, hasta que decidió casarse y se fue a vivir a España. Mientras fue soltera, Andrea tuvo libertad para disponer de su tiempo y organizar sus actividades en ambos lados de la frontera. En la Tabla 4 su trayectoria registra los continuos cambios de empleo debido a que sus pacientes fallecían, así como un cruce fronterizo frecuente, ya que por muchos años trabajó tanto en Tijuana como en San Diego, mientras vivía en Playas de Rosarito.

\section{b) De vivir en Tijuana y trabajar en San Diego, a vivir y trabajar en San Diego}

En contraste con la anterior, esta trayectoria parece ser característica de mujeres de mayor edad, con educación primaria y que trabajaron antes del matrimonio y la crianza de sus hijos, y cuando estos crecieron decidieron volver al mercado de trabajo por presiones económicas familiares debido a situaciones de enfermedad de algún familiar o desempleo del marido. Por ejemplo, Matty, quien es de Ensenada, trabajó como operadora en una maquiladora de costura cuando era joven, y volvió al mercado de trabajo debido a presiones económicas familiares. Ella empezó a trabajar cuidando a un adulto mayor en San Diego debido a que en su ciudad de origen ya no pudo conseguir empleo en el sector de la industria de la confección, en el cual había trabajado cuando era joven. Matty se retiró de este empleo para atender a su familia, sin embargo, cuando sus hijos crecieron tuvo que buscar trabajo para poder ayudar a mantener a sus padres. Si bien intentó volver a trabajar en una fábrica de costura, no pudo continuar porque sintió que se había descalificado y que no era capaz de usar las nuevas máquinas de coser. Por esa razón decidió probar suerte en el servicio doméstico, sin embargo, después de algunos meses dejó ese empleo debido a que fue objeto de acoso sexual.

Desalentada por estas experiencias, además de que su esposo enfermó y perdió el empleo, Matty decidió probar suerte en San Diego y una amiga la recomendó para cuidar a un adulto mayor que estaba discapacitado a raíz de un accidente. Inicialmente cruzaba la frontera con su visa de turista para quedarse en San Diego toda la semana y los viernes volvía a su casa para ver a su familia. Sin embargo, el control cada vez mayor sobre la frontera incrementó los tiempos de cruce y la obligó a espaciar sus viajes de regreso a Ensenada, razón por la cual sus hijas y esposo la visitaban en San Diego.

Las responsabilidades familiares interrumpieron su trayecto laboral como cuidadora, ya que Matty regresó a Ensenada para cuidar a su madre enferma. Como la señora no sanaba, Matty negoció con su hermana y ella accedió a cuidarla, y así pudo regresar a trabajar en San Diego con la misma familia. Más adelante, el enfermo que cuidaba falleció y su patrona le pidió que se fuera a cuidar a sus nietas en casa de su hija, lo cual significó un cambio en el tipo de cuidados que realizaba. Después se venció su visa y ya no pudo regresar a Ensenada. 
Tabla 5: Trayectoria transfronteriza de cuidados. Matty, 63 años, educación primaria, niñera

\begin{tabular}{|c|c|c|c|c|c|}
\hline Año & Ciudad & Ocupación & $\begin{array}{c}\text { Horario } \\
\text { de } \\
\text { trabajo }\end{array}$ & Salario & $\begin{array}{c}\text { Estatus } \\
\text { migratorio } \\
\text { y cruce }\end{array}$ \\
\hline $1988-2000$ & Ensenada & $\begin{array}{l}\text { Costurera en } \\
\text { maquiladora }\end{array}$ & $7-4$ & Mínimo & \\
\hline 2010 & Ensenada & $\begin{array}{l}\text { Costurera en } \\
\text { maquiladora }\end{array}$ & $7-4$ & Mínimo & \\
\hline 2011 & Ensenada & $\begin{array}{l}\text { Trabajadora } \\
\text { doméstica }\end{array}$ & $9-5$ & N.D. & \\
\hline 2011 & San Diego & $\begin{array}{l}\text { Cuidado de } \\
\text { adultos mayores }\end{array}$ & $\begin{array}{l}9-5 \\
\text { Lun-vie }\end{array}$ & 300 dólares & $\begin{array}{l}\text { Visa de turista } \\
\text { Lun-vie }\end{array}$ \\
\hline 2016 & San Diego & $\begin{array}{l}\text { Cuidado infantil } \\
2 \text { niñas, edades } \\
6 \text { y } 8 \text { años }\end{array}$ & $\begin{array}{l}7-5 \\
\text { Lun-vie }\end{array}$ & 350 dólares & $\begin{array}{l}\text { Indocumentada } \\
\text { Lun-vie }\end{array}$ \\
\hline
\end{tabular}

Fuente: elaboración propia con base en entrevista realizada en 2016.

Al momento de la entrevista, Matty tenía cinco años sin cruzar la frontera, así que su marido y sus hijas la visitaban en San Diego. Sin embargo, con el tiempo las visitas se fueron haciendo menos frecuentes debido a que la enfermedad de su esposo se agravó y sus hijas no siempre podían llevarlo a verla. Para seguir en contacto, la familia decidió comunicarse por Skype, además de las habituales llamadas telefónicas. Su patrona, consciente de las dificultades de la separación familiar de Matty, en las vacaciones de diciembre le prestaba un departamento del que es propietaria para que su familia pudiera estar con ella durante algunos días.

La Tabla 5 muestra la movilidad de la trayectoria laboral de Matty, primero trabajando en Ensenada en su juventud, después su inserción en el mercado informal de cuidados en San Diego; de igual manera se muestra el cambio en su movilidad transfronteriza que fue restringida por la pérdida de su visa, por lo que tuvo que quedarse a vivir en San Diego, lo cual contrasta con la alta movilidad transfronteriza que muestra la trayectoria de Andrea.

\section{Conclusiones}

Si bien el trabajo de mujeres mexicanas en Estados Unidos, y en particular en California, ha estado presente desde hace décadas, los estudios sobre el tema han dado menor atención a la dimensión transfronteriza del trabajo de cuidados, es por eso que en este artículo analizamos el caso de las trayectorias laborales de las cuidadoras y sus dinámicas de movilidad a través de la frontera Tijuana-San Diego.

A diferencia de regiones transfronterizas en el mundo que se caracterizan por una migración circular del trabajo de cuidados definida por su temporalidad, la 
transmigración como práctica de cruce cotidiano frecuente de la frontera para trabajar ha sido común en las poblaciones fronterizas del norte de México con Estados Unidos.

Las mujeres entrevistadas trabajan al otro lado de la frontera por distintas motivaciones. Las mujeres adultas ingresan a la actividad cuando sus hijos ya crecieron, para ellas es una forma de contribuir a los ingresos de la familia en situaciones imprevistas como casos de enfermedad o pérdida de empleo del marido. En el caso de las mujeres jóvenes, el trabajo de cuidados es una opción para pagar su educación, contribuir al ingreso familiar o independizarse de sus familias y, sobre todo, para obtener movilidad social a través de la educación.

Algunos estudios sobre el trabajo de cuidados en las fronteras se han enfocado en la migración circular, y dan cuenta de la precariedad y los niveles de explotación que caracteriza a la actividad. Por ejemplo, Leiva y Orellana (2016) en su estudio sobre trayectorias laborales de migrantes bolivianas en Chile destacan la hiperfragmentación de las trayectorias de las trabajadoras que se debe a las condiciones de explotación laboral. En el caso de las trabajadoras transfronterizas mexicanas usamos el concepto de transmigración, ya que la proximidad geográfica permite que las trabajadoras pueden ir y venir a través de la frontera con mayor frecuencia. Algunas de las trayectorias de las trabajadoras entrevistadas observan al inicio alta rotación laboral debido a situaciones de precariedad, sin embargo, para ellas esto puede cambiar con la experiencia, por lo cual, en trayectorias más estables, la pérdida de empleo está asociada al curso de vida de las personas a las que se cuida.

Por otra parte, los arreglos laborales de las trabajadoras están asociados a sus dinámicas de cruce, y el contexto antiinmigrante de la frontera norte de México, y de esta región específica que es Tijuana-San Diego, ha tenido consecuencias para los arreglos laborales de las trabajadoras transfronterizas de cuidados, pues las obliga a modificar sus dinámicas de cruce, por lo cual ellas optan por permanecer en su lugar de trabajo en San Diego durante la semana y regresar a Tijuana los fines de semana para estar con su familia, por lo que predominan las estrategias laborales live-in.

Por otra parte, el curso de vida y las responsabilidades familiares influyen en las trayectorias laborales de las entrevistadas, que en el caso de las mujeres jóvenes se caracterizan por su dinamismo debido a que combinan el trabajo en San Diego mientras estudian y a veces también trabajan en Tijuana. Asimismo, aspectos individuales como el estatus migratorio, la escolaridad y la experiencia en los cuidados determinan los arreglos laborales y salariales. Por ejemplo, el estatus migratorio condiciona su movilidad transfronteriza, y la escolaridad es un factor positivo ya que las mujeres con mayor nivel de educación formal obtienen mejores salarios y condiciones de trabajo que aquellas con educación primaria o educación media.

En conclusión, las experiencias de este grupo de trabajadoras transfronterizas de cuidados dan cuenta del conjunto de elementos que establecen las condiciones en las que participan en el mercado de cuidados en Estados Unidos, y expresan las complejidades de las dinámicas de cruce en función de las políticas migratorias de ese país, así como las asimetrías y desigualdades que se enuncian en las trayectorias laborales transfronterizas de las trabajadoras, pero también las estrategias que articulan para vivir y trabajar en actividades de cuidados en una región transfronteriza. 


\section{Agradecimientos}

Este trabajo se llevó a cabo durante la estancia sabática realizada en el Center for USMEX Studies, University of California en San Diego (2019-2018) con el apoyo de la Beca Sabática de Conacyt, y forma parte del proyecto "El trabajo transfronterizo de cuidados en la región Tijuana-San Diego. Profesión de vida en la intersección entre la migración, el bienestar y el género". Agradezco a los colegas del Seminario del Center for USMEX Studies, a Doreen Mattingly, así como a las personas que dictaminaron este artículo, sus comentarios y sugerencias que fueron un valioso insumo para enriquecer su contenido.

\section{Referencias}

Alber, E. \& Drotbohm, H. (Eds.). (2015). Anthropological Perspectives on Care. Work, Kinship, and the Life Course. Palgrave McMillan.

Alegría, T. (2002). Demand and Supply of Mexican Cross-border Workers. Journal of Borderlands Studies, 17(1), 37-55.

Ariza, M. (2011). Mercados de trabajo secundarios e inmigración: el servicio doméstico en Estados Unidos. Reis, 136, 3-23.

Batthyány, K. (2010). Adultos mayores, género y cuidados. Envejecimiento, género y politicas públicas. Coloquio de expertos (9 y 10 de septiembre de 2010) (pp. 91-98). Universidad de la República, Fondo de Población de las Naciones Unidas, Núcleo Interdisciplinario de Estudios sobre Vejez y Envejecimiento, Naciones Unidas Uruguay.

Benería, L. (2008). The Crisis of Care, International Migration, and Public Policy. Feminist Economics, 14(3), 1-21.

Carcedo, A., Chávez, M. J. \& Lexartza, L. (2011). Cadenas globales de cuidados: el papel de las mujeres migrantes nicaragüenses en la provisión de cuidados en Costa Rica. ONU-Mujeres.

Carrasquer, P. (2013). El redescubrimiento del trabajo de cuidados. Algunas reflexiones desde la sociología. Cuaderno de Relaciones Laborales, 31(1), 91-113.

Cienfuegos, J. (2016). Una aproximación a las desigualdades a propósito de la familia transnacional: tensiones micro y macrosociales. Desacatos, 52, 68-85.

Colen, S. (1995). "Like a Mother to Them": Stratified Reproduction and West Indian Childcare Workers and Employers in New York. En F. Ginsburg \& R. Rapp (Eds.). Conceiving the New World Order: The Global Politics of Reproduction (pp. 78102). University of California Press.

Coubès, M. L. (2008). Maquiladoras or Cross-Border Commute: The Employment of Members of Households in Five Mexican Border Cities. En R. Marquez \& H. D. Romo (Eds.), Transformations of La Familia on the U. S.-Mexico Border (pp. 131163). University of Notre Dame Press.

Coubès, M. L. \& Silva, A. (2013). Empleo, ingreso y familia. Evolución y crisis en Tijuana. En S. López (Coord.), La realidad social y las violencias. Zona metropolitana de Tijuana. El Colegio de la Frontera Norte. 
Cretu, O. (2017). Understanding Trans-Border Career Trajectories: Post-Soviet Women Professionals in London (Ph.D. Thesis). Middlesex University.

Daly, M. \& Lewis, J. (2000). The Concept of Social Care and the Analysis of Contemporary Welfare States. British Journal of Sociology, 51(2), 281-298.

Durin, S., De la O, M. E. y Bastos, S. (Coords.). (2014). Trabajadoras en la sombra. Dimensiones del servicio doméstico latinoamericano. Publicaciones de la Casa Chata, CIESAS Occidente/EGAP.

Ehrenreich, B. \& Hochschild, A. R. (2003). Global Woman. Nannies, Maids, and Sex Workers in the New Economy. Metropolitan Books.

Glaser, B. \& Strauss, A. (2012). The Discovery of Grounded Theory. Aldine Press.

Hirai, S. \& Sandoval, R. (2016). El itinerario subjetivo como herramienta de análisis: las experiencias de los jóvenes de la generación 1.5 que retornan a México. Mexican Studies/Estudios Mexicanos, 32(2), 276-301.

Hondagneu-Sotelo, P. (2007). Doméstica: Immigrant Workers Cleaning and Caring in the Shadows of Affluence. University of California Press.

Hochschild, A. (2000). Global Care Chains and Emotional Surplus Value. En T. Giddens \& W. Hutton (Eds.), On the Edge: Globalization and the New Millennium ( $p p$. 130-146). Sage Publishers.

Kofman, E. \& Raghuram, P. (2012). Women, Migration, and Care: Explorations of Diversity and Dynamism in the Global South, Social Politics, 19(3), 408-432.

Leiva, S. \& Orellana, C. R. (2016). Migración circular y trabajo de cuidado: Fragmentación de trayectorias laborales de migrantes bolivianas en Tarapacá. Psicoperspectivas. Individuo y Sociedad, 15(3), 56-66.

Leiva, S., Mansilla, M. A. \& Comelin, A. (2017). Condiciones laborales de migrantes bolivianas que realizan trabajo de cuidado en Iquique. Si Somos Americanos. Revista de Estudios Transfronterizos, 17(1), p. 11-37.

López, S. (2019). El trabajo transfronterizo de cuidados en la región Tijuana-San Diego. En M. L. Coubès, F. Uribe \& E. Vargas (Coords.), Población y salud en el nuevo escenario fronterizo del norte de México (pp. 115-144). El Colegio de la Frontera Norte.

Maher, K. H. (2003). Identity Projects at Home and Labor From Abroad: the Market for Foreign Domestic Workers in Southern California and Santiago, Chile. Working paper 75. The Center for Comparative Immigration Studies, UCSD.

Marchetti, S. (2013). Dreaming Circularity? Eastern European Women and Job Sharing in Paid Home Care. Journal of Immigrant E Refugee Studies, 11(4), 347-363. https://dx.doi.org/10.1080/15562948.2013.827770

Marcu, S. (2009). Inmigrantes rumanas en el servicio doméstico y de cuidados de la Comunidad de Madrid: Estudio cualitativo. Estudios Geográficos, 70(267), 463489. https://dx.doi.org/10.3989/estgeogr.0474

Mattingly, D. (2006). Domestic Service and International Networks of Caring Labor. En D. Mattingly \& H. Hanson (Eds.), Women and Change at the US-Mexico Border, Mobility, Labor, and Activism (pp. 103-124). Arizona University Press.

Mendoza, J. E. (2017). Economic Integration and Cross-Border Economic Organizations: The Case of San Diego-Tijuana. Estudios Fronterizos, 18(35), 22-46. https:// doi.org/10.21670/ref.2017.35.a02 
Minnesota Population Center. (2017). Integrated Public Use Microdata Series, International: Version 6.5 [dataset]. University of Minnesota. https://doi. org/10.18128/D020.V6.5

Orraca, P. (2015). Immigrants and Cross-Border Workers in the U.S.-Mexico Border Region. Frontera Norte, 27(53), 5-34.

Palenga-Möllenbeck, E. (2013). Care Chains in Eastern and Central Europe: Male and Female Domestic Work at the Intersections of Gender, Class, and Ethnicity. Journal of Immigrant $\mathcal{E}$ Refugee Studies, 11(4), 364-383. https:/ /dx.doi.org/10.1080/ 15562948.2013.822955

Parella, S., Petroff, A. \& Solé, C. (2013). The Upward Occupational Mobility of Immigrant Women in Spain. Journal of Ethnic and Migration Studies, 39(9), 1365-1382. https://dx.doi.org/10.1080/1369183X.2013.815395

Pérez-Orozco, A. (2014). Subversión feminista de la economía. Aportes para un debate sobre el conflicto capital-vida (Colección Mapas). Traficantes de sueños.

Pisani, M. \& Yoskowitz, D. (2002). The Maid Trade: Cross Border Work in South Texas. Social Science Quarterly, 83(2), 568-579.

Razavi, Sh. \& Staab, S. (2010). Underpaid and Overworked: a Cross-National Perspective on Care Workers. International Labour Review, 149(4), 407-422.

Rivera, L. (2012). Las trayectorias en los estudios de migración: una herramienta para el análisis longitudinal cualitativo. En M. Ariza \& L. Velasco (Coords.), Métodos cualitativos y su aplicación empirica. Por los caminos de la investigación sobre migración internacional (pp. 455-496). IIS-UNAM/El Colef.

Sassen, S. (2003). Contrageografías de la globalización. Género y ciudadanía en los circuitos transfronterizos (Colección Mapas). Traficantes de Sueños.

Schulman, K. \& Blank, H. (2017). Persistent Gaps: State Child Care Assistance Policies 2017. National Women's Law Center.

Soto, C., González, M. \& Dobrée, P. (2012). La migración femenina paraguaya en las cadenas globales de cuidados en Argentina. Transferencias de cuidado y desigualdades de género. ONU-Mujeres.

Truong, T. D. (1996). Gender, International Migration, and Social Reproduction: Implications for Theory, Social Research, and Networking. Asian and Pacific Migration Journal, 5(1), 27-52.

Williams, F. (2011). Towards a transnational analysis of the political economy of care. En F. Williams \& F. Robinson (Eds.), Feminist ethics and social policy. Toward a new global political economy of care (pp. 21-39). UBC Press/The University of British Columbia.

Yeates, N. (2005). Global Care Chains: a Critical Introduction. Global Migration Perspectives, 44.

Yeates, N. (2009). Globalizing Care Economies and Migrant Workers: Explorations in Global Care Chains. Palgrave. 
Silvia López Estrada

Mexicana. Doctora en sociología por The City University of New York, Graduate Center; profesora-investigadora Titular C, Departamento de Estudios de Población, El Colegio de la Frontera Norte. Sus principales líneas de investigación son: trabajo, familia y género; trabajo, cuidados y género; políticas públicas y género. Entre sus publicaciones recientes están: Guadarrama, R., Hualde, A. \& López, S. (2014). La precariedad laboral en México. Dimensiones, dinámicas y significados. El Colef/uam; y López, S. (Coord.). (2017). Género y políticas públicas. Retos y oportunidades para la transversalidadad en Nuevo León. El Colef. 